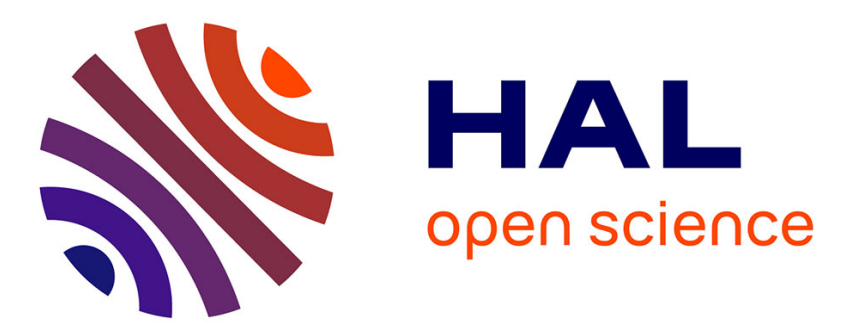

\title{
Effect of environmental fluctuations on invasion fronts
}

\author{
Vicenç Méndez, Isaac Llopis, Daniel Campos, Werner Horsthemke
}

\section{To cite this version:}

Vicenç Méndez, Isaac Llopis, Daniel Campos, Werner Horsthemke. Effect of environmental fluctuations on invasion fronts. Journal of Theoretical Biology, 2011, 281 (1), pp.31. 10.1016/j.jtbi.2011.04.025 . hal-00708522

\section{HAL Id: hal-00708522 \\ https://hal.science/hal-00708522}

Submitted on 15 Jun 2012

HAL is a multi-disciplinary open access archive for the deposit and dissemination of scientific research documents, whether they are published or not. The documents may come from teaching and research institutions in France or abroad, or from public or private research centers.
L'archive ouverte pluridisciplinaire HAL, est destinée au dépôt et à la diffusion de documents scientifiques de niveau recherche, publiés ou non, émanant des établissements d'enseignement et de recherche français ou étrangers, des laboratoires publics ou privés. 


\section{Author's Accepted Manuscript}

Effect of environmental fluctuations on invasion fronts

Vicenç Méndez, Isaac Llopis, Daniel Campos, Werner Horsthemke

$\begin{array}{ll}\text { PII: } & \text { S0022-5193(11)00225-6 } \\ \text { DOI: } & \text { doi:10.1016/j.jtbi.2011.04.025 } \\ \text { Reference: } & \text { YJTBI 6456 }\end{array}$

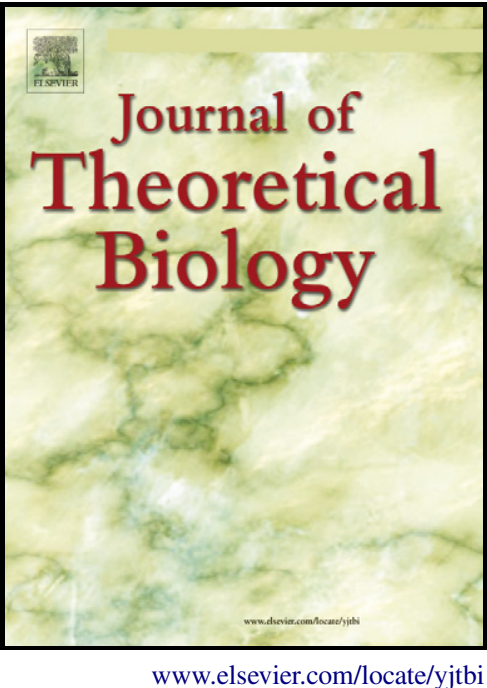

To appear in: $\quad$ Journal of Theoretical Biology

Received date: 21 September 2010

Revised date: $\quad 15$ March 2011

Accepted date: 23 April 2011

Cite this article as: Vicenç Méndez, Isaac Llopis, Daniel Campos and Werner Horsthemke, Effect of environmental fluctuations on invasion fronts, Journal of Theoretical Biology, doi:10.1016/j.jtbi.2011.04.025

This is a PDF file of an unedited manuscript that has been accepted for publication. As a service to our customers we are providing this early version of the manuscript. The manuscript will undergo copyediting, typesetting, and review of the resulting galley proof before it is published in its final citable form. Please note that during the production process errors may be discovered which could affect the content, and all legal disclaimers that apply to the journal pertain. 


\section{Effect of environmental fluctuations on invasion fronts}

Vicenç Méndez ${ }^{\mathrm{a}, *}$, Isaac Llopis ${ }^{\mathrm{b}}$, Daniel Campos ${ }^{\mathrm{a}}$ and Werner Horsthemke ${ }^{\mathrm{c}}$

${ }^{a}$ Grup de Física Estadística. Departament de Física. Universitat Autònoma de Barcelona, 08193 Bellaterra (Barcelona) Spain

${ }^{\mathrm{b}}$ School of Mathematics and Statistics, Newcastle University, Newcastle Upon Tyne, NE1 $7 R U, U K$

${ }^{\mathrm{c}}$ Department of Chemistry, Southern Methodist University, Dallas, Texas 75275-0314, USA 


\section{Abstract}

We determine the density profile and velocity of invasion fronts in one-dimensional infinite habitats in the presence of environmental fluctuations. The population dynamics is reformulated in terms of a stochastic reaction-diffusion equation and is reduced to a deterministic equation that incorporates the systematic contributions of the noise. We obtain analytical expressions for the front profile and velocity by constructing a variational principle. The effect of the noise differs, depending on whether it affects the density-independent growth rate, the intraspecific competition term or the Allee threshold. Fluctuations in the density-independent growth rate increase the invasion velocity and the population density of the invaded area. Fluctuations in the competition term also change the population density of the invaded area, but modify the invasion velocity only for certain initial conditions. Fluctuations in the Allee threshold can induce pulled or pushed invasion fronts as well as invasion failure. We compare our analytical results with numerical solutions of the stochastic partial differential equations and show that our procedure proves useful in dealing with reaction-diffusion equations with multiplicative noise.

Key words: Invasion front, Environmental fluctuations, Allee effect

\footnotetext{
* Corresponding author.

Email address: vicenc.mendez@uab.es (Vicenç Méndez).
} 


\section{INTRODUCTION}

Biological invasions correspond to the introduction and spread of exotic organisms into regions outside of their native range. They have emerged as a major environmental, economic and public health problem tied to the rapid, ongoing expansion of international trade and travel and have been studied for many decades (Fisher, 1937; Kolmogorov et al, 1937; Skellam, 1951; Hengeveld, 1989; Williamson, 1996; Shigesada and Kawasaki, 1997; Murray, 2003). The invasion process can be regarded as a traveling wave front that propagates through a homogeneous medium, and it can be described mathematically by reactiondiffusion equations (Shigesada and Kawasaki, 1997; Murray, 2003; OrtegaCejas et al., 2004; Méndez et al., 2010). These equations can be generalized to take into account memory effects in the animal motion for logistic dynamics (Ortega-Cejas et al. 2004) and dynamics with Allee effect (Méndez and Compte, 1998).

Another important topic in ecology is the role of fluctuations in population dynamics (Horsthemke and Lefever, 1984). Populations with a small number of individuals experience internal fluctuations and require a stochastic description. Even if these fluctuations can be neglected because the population contains a large number of individuals, other stochastic factors are usually present and can affect significantly the dynamic behavior of the population. Examples are demographic stochasticity, environmental stochasticity, demographic heterogeneity and stochastic sex determination. Various authors have investigated the effect of random fluctuations on population dynamics. Marcus studied a stochastic partial differential equation (SPDE), namely a reaction-diffusion equation with logistic growth and multiplicative spatiotem- 
poral Gaussian white noise (Marcus, 1982). He carried out a small noise expansion and established the existence and uniqueness of a weak solution of the SPDE. More recently, Malchow and coworkers have studied numerically the effect of external noise on pattern formation in reaction-diffusion models of plankton dynamics (Malchow et al, 2004a; Malchow et al, 2004b). Scarsoglio and coworkers have used analytical and numerical tools to investigate the effects of external fluctuations on vegetation patterns (Scarsoglio et al., 2011).

Already Skellam (1951) analyzed the effects of random dispersal on population spread and applied his results to the spread of oak in Britain and the muskrat in Central Europe. Mollison (1991) used linear stochastic models to estimate invasion velocities. The author stressed the limitations of linear models and the need for a better understanding of nonlinear stochastic models. Lewis similarly stressed the importance of stochastic factors for biological invasions (Lewis, 2000) and used a discrete-time continuous-space model, an integrodifference equation, to study the effect of intrinsic stochastic factors. Deriving equations for the first two spatial moments of the population density via moment closure approximations, he obtained an upper bound for the invasion speed via comparison methods. Neubert and Caswell (2000) combined integrodifference equations for dispersal with matrix models for population growth to analyze invasion velocity in populations with stage structure. Though they did not incorporate stochastic effects in their description, they acknowledged the importance of including environmental stochasticity and demographic stochasticity in their discussion of directions for future research. They also acknowledged the importance of including Allee effects (Allee, 1938). The issue of environmental stochasticity has been addressed by Schreiber and 
Ryan, who studied the effect of a fluctuating environment in integrodifference matrix models (Schreiber and Ryan, 2011). Melbourne and Hastings have reported experimental studies to assess the effects of various stochastic factors on extinction risk (Melbourne and Hastings, 2008) and the effects of demographic stochasticity on spread rates in populations for the red flour beetle (Tribolium castaneum) (Melbourne and Hastings, 2009).

Here we investigate the effect of environmental stochasticity on the invasion velocity of two types of population dynamics, namely logistic growth and growth with Allee effect. For logistic growth, we consider an external noise that gives rise to fluctuations in either the density-independent growth rate or the competition term. For growth with Allee effect, we consider an external noise that gives rise to fluctuations in the Allee threshold. This requires us to address the open problem of how to include stochasticity in an analytically tractable model as stated by different authors (see, e.g., Mollison, 1991; Melbourne and Hastings, 2008).

We adopt a continuous-time continuous-space description of the population, namely reaction-diffusion equations with multiplicative, spatially correlated, temporally white Gaussian noise. The effect of external noise on propagating fronts has been studied in physical and chemical reaction-diffusion systems, mostly for the case of bistable and excitable systems (see, e.g., SchimanskyGeier and Zülicke, 1991; Armero et al., 1996; García-Ojalvo and Sancho, 1999; Hizanidis et al., 2006). Our approach consists in decomposing the population density into a systematic (or deterministic) part and a fluctuating part. We derive analytically the reaction-diffusion equation for the deterministic part and construct a variational principle that provides the invasion velocity and the front profile by maximizing a functional. This allows us to study analyti- 
cally the effect of environmental stochasticity on the invasion velocity and on the front profile.

We find for logistic growth that if fluctuations are present in the densityindependent growth rate, then the final state after invasion experiences an increase in the population density and the invasion velocity increases with the intensity of fluctuations. If fluctuations affect the competition term, then two types of invasion fronts can occur. The first type corresponds to the front observed in the deterministic system. It occurs for certain initial conditions. The population density of the final stable state after invasion increases in the presence of fluctuations. The invasion front, however, travels with the Fisher velocity, that is, the invasion velocity does not depend on the intensity of fluctuations. For other initial conditions, and if the intensity of the fluctuations is low enough, a noise-induced front occurs that corresponds to the collapse of a noise-induced high-density state to the effective carrying capacity of the system. This front travels with a velocity that decreases with the intensity of the fluctuations. In the cases mentioned above, the front is pulled-its dynamics is governed by the tail of the front-in the absence of fluctuations and remains pulled in the presence of fluctuations. For growth with strong Allee effect, fluctuations in the Allee threshold give rise to a richer behavior. In this case, the invasion front is pushed-its dynamics is governed by the interior part of the front-in the absence of fluctuations. In their presence, the invasion front can be pulled, pushed or even disappear all together, depending on the intensity of the fluctuations. 


\section{EQUATION FOR THE SYSTEMATIC POPULATION DEN- SITY}

Consider a population with density $\phi(x, t)$. For simplicity, we assume that the population lives in a one dimensional system and evolves dynamically according to a reaction-diffusion equation,

$$
\frac{\partial \phi(x, t)}{\partial t}=D \frac{\partial^{2} \phi(x, t)}{\partial x^{2}}+F[\phi(x, t), t]
$$

We consider two types of population dynamics, i.e., two forms of $F(\phi)$, namely logistic population growth,

$$
F(\phi, t)=r_{1} \phi(x, t)-r_{2} \phi(x, t)^{2}
$$

and growth with Allee effect,

$$
F(\phi, t)=r \phi(1-\phi)(\phi-a)
$$

For logistic growth, $r_{1}$ is the density-independent growth rate, or the coefficient of increase to use Skellam's terminology (Skellam, 1951). The coefficient $r_{2}$ is the limiting coefficient in Skellam's terminology; it describes intraspecific competition or the struggle for resources. For growth with Allee effect, $r$ is the growth rate and $a$ the Allee threshold. We consider only the case $a>0$, i.e., a strong Allee effect. For logistic growth, one usually writes $r_{1}=r$ and $r_{2}=r / K$, where $K$ is the carrying capacity of the environment. In general, we can consider these parameters to be functions of space and time, e.g., $r_{1}(x, t)$ and $r_{2}(x, t)$, though they are usually treated as constants in the literature (Murray, 2003). Equation (1) does not include fluctuations explicitly. Environmental fluctuations, i.e., external noise, turns the parameters $r, K$ and $a$ into stochastic quantities and (1) into a SPDE, whose solution will be a 
functional of the noise (San Miguel and Toral, 2000),

$$
\frac{\partial \phi}{\partial t}=D \frac{\partial^{2} \phi}{\partial x^{2}}+F(\phi)+\varepsilon^{1 / 2} g(\phi) \eta(x, t)
$$

where $\eta(x, t)$ represents the noise and $\varepsilon$ measures the noise strength. Note that the external noise appears in a multiplicative way in (4) if $g(\phi)$ is not constant. Motivated by the Central Limit Theorem, we consider the case that the noise is Gaussian with zero mean, $\langle\eta(x, t)\rangle=0$. We further assume that the fluctuations occur on a faster time scale than that of the systematic population growth. This implies that we can consider the noise to be white in time, i.e., it has a correlation function given by

$$
\left\langle\eta(x, t) \eta\left(x^{\prime}, t^{\prime}\right)\right\rangle=2 C\left(\left|x-x^{\prime}\right| / \ell\right) \delta\left(t-t^{\prime}\right)
$$

Here $C(x)$ is the spatial correlation function of the noise and $\ell$ its characteristic length. In the numerical simulations $C(x)$ has been chosen to be Gaussian with variance 1 and $C(0)=10$. Since the noise is white in time, the population density is a Markovian stochastic variable.

The mean value of the noise term $g(\phi) \eta(x, t)$ does not vanish if $g(\phi)$ is not constant, which gives rise to a systematic contribution to the dynamics of the density (García-Ojalvo and Sancho, 1999). Adopting the Stratonovich interpretation of the SPDE (4), we evaluate $\langle g(\phi) \eta(x, t)\rangle$ using Novikov's theorem (Novikov, 1965), see Appendix A for details,

$$
\langle g(\phi) \eta(x, t)\rangle=\varepsilon^{1 / 2} C(0)\left\langle\frac{d g(\phi)}{d \phi} g(\phi)\right\rangle .
$$

This result allows us to rewrite (4) in a form where the noise term has zero mean,

$$
\frac{\partial \phi}{\partial t}=D \frac{\partial^{2} \phi}{\partial x^{2}}+F_{\mathrm{eff}}(\phi)+\varepsilon^{1 / 2} \zeta(\phi, x, t)
$$


The effective reaction term and the new noise term are given by

$$
F_{\mathrm{eff}}(\phi)=F(\phi)+\varepsilon C(0) \frac{d g(\phi)}{d \phi} g(\phi)
$$

and

$$
\zeta(\phi, x, t)=g(\phi) \eta(x, t)-\varepsilon^{1 / 2} C(0) \frac{d g(\phi)}{d \phi} g(\phi),
$$

respectively. We decompose the population density into a systematic, or deterministic, part and a fluctuating part by writing the density as

$$
\phi(x, t)=\rho(x, t)+\sum_{n=1}^{\infty} \varepsilon^{n / 2} \rho_{n}(x, t) .
$$

The first term $\rho(x, t)$ is deterministic and includes the systematic contributions. The sum represents the fluctuating part of the density. This expansion represents an extension of the standard small-noise expansion procedure (see, e.g., Marcus 1982); the form (10) keeps the relevant, systematic contributions of the multiplicative noise. It allows us to separate the systematic effects from the purely stochastic effects of the external noise on the population density. We assume that the deterministic contribution dominates, while the stochastic part plays only a small role. We will show by comparing the analytical results with numerical simulations that focusing solely on the deterministic density represents an excellent approximation.

Substitution of (10) into (7) yields to the lowest order the equation for the systematic (deterministic) contribution,

$$
\frac{\partial \rho}{\partial t}=D \frac{\partial^{2} \rho}{\partial x^{2}}+F_{\mathrm{eff}}(\rho)
$$

The equation for the next order, the first stochastic contribution, is given by

$$
\frac{\partial \rho_{1}}{\partial t}=D \frac{\partial^{2} \rho_{1}}{\partial x^{2}}+\frac{d F_{\text {eff }}(\rho)}{d \rho} \rho_{1}+\zeta(\rho, x, t)
$$


By construction, see (9), the mean value of the noise $\zeta(\rho, x, t)$ is zero, and its correlation function is given by

$$
\left\langle\zeta(\rho, x, t) \zeta\left(\rho, x^{\prime}, t^{\prime}\right)\right\rangle=g(\rho(x, t)) g\left(\rho\left(x^{\prime}, t^{\prime}\right)\right)\left\langle\eta(x, t) \eta\left(x^{\prime}, t^{\prime}\right)\right\rangle .
$$

The equation for the systematic density, the dominant contribution, can be written as

$$
\frac{\partial \rho}{\partial t}=D \frac{\partial^{2} \rho}{\partial x^{2}}+F(\rho)+\varepsilon_{0} \frac{d g(\rho)}{d \rho} g(\rho)
$$

where $\varepsilon_{0} \equiv \varepsilon C(0)$.

We investigate this equation explicitly for three cases: (i) fluctuations in the density-independent growth rate, (ii) fluctuations in the carrying capacity, and (iii) fluctuations in the Allee threshold. Stochastic variations in environmental variables, such as temperature and precipitation for example, lead to random changes in the vital rates of populations (Schreiber and Ryan, 2011). Fluctuations in the density-independent growth rate (Tuckwell, 1974) occur because the difference between births and deaths changes with time and fluctuates around a mean value, due to external stochastic factors. Fluctuations in the competition term represent temporal variations in the availability of resources (Feldman and Roughgarden, 1975). Finally, fluctuations in the Allee threshold occur because the minimum number of individuals necessary for the population to grow fluctuates in space and time due to environmental factors.

\subsection{Fluctuating density-independent growth rate}

If the density-independent growth rate fluctuates around a mean value $r_{m}$, we write $r_{1}(x, t)=r_{m}\left[1+\varepsilon^{1 / 2} \eta(x, t)\right]$ and $r_{2}=r_{m} / K$, with $r_{m}$ and $K$ constants. Introducing the expression for the fluctuations of the density-independent 
growth rate into (1), we obtain the following SPDE:

$$
\frac{\partial \phi}{\partial t}=D \frac{\partial^{2} \phi}{\partial x^{2}}+r_{m} \phi\left(1-\frac{\phi}{K}\right)+\varepsilon^{1 / 2} r_{m} \phi \eta(x, t)
$$

In this case $g(\phi)=r_{m} \phi$, as can be seen by comparing (15) and (4). According to (14), the systematic density obeys the reaction-diffusion equation

$$
\frac{\partial \rho}{\partial t}=D \frac{\partial^{2} \rho}{\partial x^{2}}+r_{m}\left(1+r_{m} \varepsilon_{0}\right) \rho-\frac{r_{m}}{K} \rho^{2}
$$

The systematic effects of the fluctuating net growth rate are represented by the term $r_{m}^{2} \varepsilon_{0} \rho$. In the following we assume $K=1$ for simplicity. Note that fluctuations in the density-independent growth rate lead to a larger effective coefficient of increase.

\subsection{Fluctuating competition term}

To model fluctuations in the carrying capacity, we consider $(2)$ with $r_{2}(x, t)=$ $\frac{r}{K_{m}}\left[1+\varepsilon^{1 / 2} \eta(x, t)\right]$ and $r_{1}(x, t)=r$. Equation (1) turns into the SPDE

$$
\frac{\partial \phi}{\partial t}=D \frac{\partial^{2} \phi}{\partial x^{2}}+r \phi\left(1-\frac{\phi}{K_{m}}\right)-\frac{r}{K_{m}} \varepsilon^{1 / 2} \phi^{2} \eta(x, t) .
$$

In this case $g(\phi)=-r \phi^{2} / K_{m}$. According to (14), we obtain the following equation for the systematic population density,

$$
\frac{\partial \rho}{\partial t}=D \frac{\partial^{2} \rho}{\partial x^{2}}+r \rho\left(1-\frac{\rho}{K_{m}}\right)+\frac{2 r^{2}}{K_{m}^{2}} \varepsilon_{0} \rho^{3}
$$

Note that noise in the competition term introduces a singular perturbation in the logistic growth model. The reaction function of the logistic model is quadratic, while the systematic contributions of the noise give rise to a cubic term in the effective reaction function. In other words, noise in the limiting term has the effect of introducing a mutualistic term due to its Gaussian 
nature. For densities above a critical value, see below, this cubic term will overcome the quadratic competition term and lead to unbounded growth. In actual populations, higher-order limiting term, e.g., terms of order $\phi^{4}$, will come into play at these high densities and prevent a population explosion. Consequently, the range of validity of (18) is restricted to densities below the critical value and to sufficiently small noise strengths.

This does not affect our studies, since the expansion (10) is essentially a smallnoise expansion and self-consistency requires sufficiently small noise strengths. In the following we assume $K_{m}=1$ for simplicity.

\subsection{Fluctuating Allee threshold}

If the Allee threshold fluctuates around the mean value $a_{m}$, we write $a(x, t)=$

$a_{m}\left[1+\varepsilon^{1 / 2} \eta(x, t)\right], r_{1}(x, t)=r_{2}(x, t)=r$ and $K=1$ and obtain the SPDE

$$
\frac{\partial \phi}{\partial t}=D \frac{\partial^{2} \phi}{\partial x^{2}}+r \phi(1-\phi)\left(\phi-a_{m}\right)-r a_{m} \varepsilon^{1 / 2} \phi(1-\phi) \eta(x, t) .
$$

Here $g(\phi)=-r a_{m} \phi(1-\phi)$, and according to (14) we find

$$
\frac{\partial \rho}{\partial t}=D \frac{\partial^{2} \rho}{\partial x^{2}}+r \rho(1-\rho)\left[\left(1-2 r \varepsilon_{0} a_{m}^{2}\right) \rho-a_{m}\left(1-r \varepsilon_{0} a_{m}\right)\right]
$$

Note that the third factor of the effective reaction term is very similar to the case without noise $\left(\varepsilon_{0}=0\right)$ but with different coefficients, which can be either positive or negative giving rise a wide range of different front solutions. In other words, external noise can decrease the effective Allee threshold and even render it negative, i.e., turn a strong Allee effect into a weak Allee effect. 


\section{INVASION FRONTS}

Consider the situation that the reaction term $F(\phi)$ in (1) has two steady states, $F(\phi)=0$, given by $\phi_{u}=0$ (unstable) and $\phi_{s}=1$ (stable). The velocity of the invasion front joining states 0 and 1 can be obtained from the variational formula,

$$
v^{2}=2 D \max _{0<\lambda \leq 2}\left[\frac{\lambda \Gamma(4)}{\Gamma(2-\lambda) \Gamma(2+\lambda)} \int_{0}^{1} F(\rho) \rho^{-\lambda}(1-\rho)^{\lambda} d \rho\right],
$$

where $\lambda$ parameterizes the set of trial functions $\left\{\rho^{-\lambda}(1-\rho)^{\lambda}\right\}$. See Appendix B for details of the derivation of the variational formula (21).

\subsection{Invasion velocities}

In the following, we determine the invasion velocity for the three cases of external noise described in Sect. 2. To do so, we apply the variational formula (21) to the evolution equation for the systematic part of the density, namely (16), (18) and (20), replacing $F(\phi)$ by $F_{\text {eff }}(\rho)$ and renormalizing the density where necessary, so that the steady states are given by 0 and 1 . We also compare our analytical results with numerical calculations. Details of the numerical algorithm to calculate the invasion velocities and the front profile are provided in Appendix C. In the following sections we find fronts invading both unstable and metastable states. The main difference is that in the first case the front velocity depends on the tail of the initial condition and in the second case the front velocity is independent. For more information about pulled and pushed fronts and fronts propagating into unstable and metastable states see the works by van Saarloos, 2003 and by Méndez et al., 2010. 


\subsubsection{Fluctuating density-independent growth rate}

If the density-independent growth rate fluctuates, the effective reaction term is given by $F_{\text {eff }}(\rho)=r_{m}\left(1+r_{m} \varepsilon_{0}\right) \rho-r_{m} \rho^{2}$ according to (16). The effective reaction term has two steady states, namely $\rho=0$ and $\rho=1+r_{m} \varepsilon_{0}$. The first steady state is unstable to small density perturbations, while the second one is stable. To be able to apply (21) we introduce the renormalized density $u=\rho /\left(1+r_{m} \varepsilon_{0}\right)$. This change of variables turns (16) into the Fisher equation with a rate constant given by $r_{m}\left(1+r_{m} \varepsilon_{0}\right)$. Consequently, the front velocity is given by

$$
v=2 \sqrt{D r_{m}\left(r_{m} \varepsilon_{0}+1\right)}
$$

We confirm this result by numerical integration of (1) and (2), see Figure 1. The velocity grows sublinearly with the stochastic noise strength as $\varepsilon^{1 / 2}$ recall that $\varepsilon_{0}=\varepsilon C(0)$. Moreover, the velocity becomes the Fisher front velocity, $v_{F}$, for $\varepsilon \rightarrow 0$, as expected.

\subsubsection{Fluctuating competition term}

If the carrying capacity fluctuates, the effective reaction term is given by $F_{\text {eff }}(\rho)=r \rho-r \rho^{2}+2 r^{2} \varepsilon_{0} \rho^{3}$ according to (18). This reaction term possesses three different steady states: $\rho=0$ and

$$
\rho_{ \pm}=\frac{1 \pm \sqrt{1-8 \varepsilon_{0} r}}{4 \varepsilon_{0} r}
$$

The nontrivial solutions, $\rho_{ \pm}$, exist only if $\varepsilon_{0}<1 / 8 r$. This is a reflection of the fact that the validity of (18) is restricted to the regime of small noise strength, as discussed in Sect. 2.2. The state $\rho_{-}$is stable, and the states 0 and $\rho_{+}$are unstable. The latter is the critical value of the population density, see Sect. 2.2, above which the population will grow without bound. Writing the 
square root as a power series, we find that

$$
\begin{aligned}
& \rho_{-}=1+\varepsilon_{0} r / 4+O\left(\varepsilon_{0}^{2}\right), \\
& \rho_{+}=\frac{1}{2 \varepsilon_{0} r}-1+O\left(\varepsilon_{0}\right) .
\end{aligned}
$$

The state $\rho_{-}$correspond to the deterministic case of the population being at the carrying capacity of the system, $\phi=K_{m}=1$. Noise in the competition term increases the effective carrying capacity of the system. The other state, $\rho_{+}$, is a pure noise-induced state. Its origin lies in the fact that noise in the competition term, i.e., in the limiting coefficient, gives rise to a systematic effect of the opposite nature, see Sect. 2.2.

We exclude here situations where the initial densities are larger than $\rho_{+}$, i.e., we exclude all cases of unbounded population growth. Then there are two possible pulled fronts propagating into unstable states, one invading the state 0 and connecting it to the state $\rho_{-}$and another invading the state $\rho_{+}$and connecting it to $\rho_{-}$. To obtain both fronts, one has to prepare the system with appropriate initial conditions. Below we indicate which initial condition we employed. Equation (21) allows us to calculate the front velocities of the two different fronts, and we compare the results with numerical results.

3.1.2.1 Pulled front connecting 0 to $\rho_{-}$. This case corresponds to the one that occurs in the deterministic system, (1) with (2). Since the noise increases the effective carrying capacity, we must again renormalize the density, $u=\rho / \rho_{-}$, to be able to apply the variational formula $(21)$. The front velocity is calculated from

$$
v^{2}=4 D r^{2} \varepsilon_{0} \rho_{+} \rho_{-} \max _{0<\lambda \leq 2}\left\{\lambda\left[1-\frac{1}{4} \frac{\rho_{-}}{\rho_{+}}(2-\lambda)\right]\right\} .
$$


Since the function to be maximized is monotonically increasing with $\lambda$, the maximum is attained at $\lambda=2$, which results in the Fisher velocity, $v_{F}=$ $2 \sqrt{D r}$. Note that in this case the front velocity does not depend on the noise strength $\varepsilon_{0}$. This result is depicted in Figure 2a, where we compare our analytical result with results from numerical simulations. For the numerical calculations we chose the initial conditions to be $\rho(x, 0)=\rho_{-}\left[1-\theta\left(x-x_{0}\right)\right]$, where $\theta(\cdot)$ is the Heaviside function and $x_{0}>0$.

\subsubsection{Pulled front connecting $\rho_{+}$to $\rho_{-}$. Such a front can occur} only in the presence of external noise; it has no deterministic counterpart. To obtain the front velocity using (21), we apply the change of variables $u=$ $\left(\rho_{+}-\rho\right) /\left(\rho_{+}-\rho_{-}\right)$and find

$$
v^{2}=4 D r^{2} \varepsilon_{0}\left(\rho_{+}-\rho_{-}\right) \rho_{+} \max _{0<\lambda \leq 2}\left\{\lambda\left[1-\frac{1}{4} \frac{\rho_{+}-\rho_{-}}{\rho_{+}}(2-\lambda)\right]\right\} .
$$

Again the function of $\lambda$ is monotonically increasing, the maximum is at $\lambda=2$, and the front velocity expression reads,

$$
v=\sqrt{\frac{D}{\varepsilon_{0}}} \sqrt{1-8 \varepsilon_{0} r+\sqrt{1-8 \varepsilon_{0} r}} .
$$

This front velocity decreases with the noise amplitude as $\varepsilon^{-1 / 2}$ and is valid only if $\varepsilon_{0}<1 / 8 r$. This result is depicted in Figure $2 \mathrm{~b}$, where we compare our analytical result with results from numerical simulations. For the numerical calculations we chose the initial conditions to be $\rho(x, 0)=\rho_{-}+\left(\rho_{+}-\rho_{-}\right) \theta(x-$ $x_{0}$ ) with $x_{0}=10$. The front corresponds to a collapse of the hight-density state $\rho_{+}$to the "deterministic" state $\rho_{-}$. The fact that the velocity decreases with the noise amplitude reflects the nature of the state $\rho_{+}$. It is a noise-induced or noise-sustained state, and its survival time increases with increasing noise strength. 


\subsubsection{Fluctuating Allee threshold}

Applying (21) to (20) we obtain

$$
v^{2}=2 D r_{m} \max _{0<\lambda \leq 2}\left[\frac{1-2 r \varepsilon_{0} a_{m}^{2}}{4} \lambda(2-\lambda)+a_{m}\left(r \varepsilon_{0} a_{m}-1\right) \lambda\right] .
$$

The maximum must be determined carefully because it depends strongly on the values for the parameters $a_{m}$ and $\varepsilon_{0}$. We can distinguish up to five different situations, which correspond to different regions of the parameter space $\left(r \varepsilon_{0}\right.$ versus $a_{m}$ ), see Figure 3. (The first two situations are shown as one region, Region I, in the figure.)

\subsubsection{Region I. Front propagating into the unstable state $\rho=0$} and connecting $\rho=0$ to $\rho=1$. In this case, the front can be pulled (the front dynamics is governed by the leading edge, i.e., the nonlinear term is not important) or pushed (i.e. the front dynamics is governed by its interior part and nonlinear term becomes more important than linear term) and can occur only for $a_{m}<1 / 2$. Using the variational formula (29) we can determine analytically where this transition takes place. Equation (29) implies that

$$
v_{I}=\left\{\begin{array}{c}
\sqrt{\frac{r D}{2} \frac{1-2 a_{m}}{\sqrt{1-2 r \varepsilon_{0} a_{m}^{2}}}}, \quad \frac{1}{a_{m}}<\varepsilon_{0} r \leq \frac{1+2 a_{m}}{4 a_{m}^{2}} \text { (pushed) }, \\
2 \sqrt{D r a_{m}\left(a_{m} r \varepsilon_{0}-1\right)}, \frac{1+2 a_{m}}{4 a_{m}^{2}} \leq \varepsilon_{0} r<\frac{1}{2 a_{m}^{2}} \text { (pulled). }
\end{array}\right.
$$

In Figure 4 we plot the invasion velocity versus $r \varepsilon_{0}$ (30) (solid line) and compare it with results from numerical solutions (circles). The transition between the pulled and pushed regime is located at $r \varepsilon_{0}=\left(1+2 a_{m}\right) /\left(4 a_{m}^{2}\right)$.

\subsubsection{Region II. Front propagating into the unstable state $\rho=0$} and connecting $\rho=0$ to $\rho^{*}=a_{m}\left(r \varepsilon_{0} a_{m}-1\right) /\left(2 r \varepsilon_{0} a_{m}^{2}-1\right)$. Note the $\rho^{*}$ is 
the effective Allee threshold in the presence of external noise. We again need to renormalize the density, $u=\rho / \rho^{*}$. In this case, the front is always pulled and the region is defined by $\varepsilon_{0} r>1 / 2 a_{m}^{2}$ if $a_{m}<1 / 2$ or by $\varepsilon_{0} r>1 / a_{m}$ if $a_{m}>1 / 2$. According to (29), the velocity of the front is given by

$$
v_{I I}=2 \sqrt{\operatorname{Dra}_{m}\left(a_{m} r \varepsilon_{0}-1\right)} .
$$

\subsubsection{Region III. Front propagating into the metastable state} $\rho=0$ and connecting $\rho=0$ to $\rho=1$. This region is defined by $\varepsilon_{0} r<1 / a_{m}$ for $a_{m}<1 / 2$. In this case, the front is always pushed and it travels with the velocity

$$
v_{I I I}=\sqrt{\frac{r D}{2}} \frac{1-2 a_{m}}{\sqrt{1-2 r \varepsilon_{0} a_{m}^{2}}} .
$$

If $\varepsilon_{0} r>1 / a_{m}$ and $a_{m}<1 / 2$ there is no front. In Figure 3 we depict these regions and compare our theoretical results with numerical simulations.

To construct numerically the region boundaries we fix $a_{m}$ and then vary $r \varepsilon_{0}$. For each value of $r \varepsilon_{0}$ we compute numerically the front profile and check in which region we are. For example, to find the boundary between regions I and II in Figure 3 we begin with a high value of $r \varepsilon_{0}$ (belonging to region I) and determine if the front velocity depends on the shape of initial condition. To this end we take $\rho(x, 0)=1-\theta(x) e^{\mu x}$ and observe that the front velocity depends explicitly on $\mu$. This indicates that the front invades the unstable state $\rho=0$ and we are in region I. When we decrease progressively $r \varepsilon_{0}$ and it crosses the boundary to enter in region III, the front velocity ceases to depend on $\mu$ which indicates that the front invades the metastable state $\rho=0$ and we are in region III. To compute the boundary between regions I and II we must observe which is the final stable state, since in both regions the front 
invades the unstable state $\rho=0$. To this end, we take the initial condition $\rho(x, 0)=0.1[1-\theta(x)]$. If the stable state (state behind the front) is $\rho=1$ then we are in region I. Otherwise, we are in region II. Finally, the boundary between region II is trivial to find numerically because below the boundary no front is formed.

\subsection{Front profiles}

Besides the front velocity, front profiles are also of interest. The variational principle allows us also to predict the profile shape, i.e., the analytical expression for $\rho(x, t)$, when the front is fully developed and the velocity has reached its stationary value. If the trial function is chosen to be $g(\rho)=\rho^{-\lambda}(1-\rho)^{\lambda}$, and using the fact that (see Appendix B)

$$
-\frac{d}{d \rho} \ln g=\frac{v}{D p}
$$

we find that

$$
p=\frac{v}{\lambda D} \rho(1-\rho) \equiv-\frac{d \rho}{d z} .
$$

Integrating over $z$, we obtain the expression for the density

$$
\rho(z)=\frac{1}{1+b^{-1} \exp \left(-\frac{v z}{\lambda D}\right)},
$$

which is a sigmoidal function. The integration constant is $b^{-1}=\left(1-\rho_{*}\right) / \rho_{*}$, where $\rho_{*}$ is the density at $z=0$. The asymptotic front profile given by (35) is the same for each of the three cases we have studied. To specify the solution for each case, we must substitute the value of $\lambda$ for which the maximum in (21) is attained and the corresponding velocity $v$. For example, when the densityindependent growth rate fluctuates, the velocity is given by (22). The density 
profile reads

$$
\rho(x, t)=\frac{1}{1+b^{-1} \exp \left(-\sqrt{\frac{r_{m}\left(1+r_{m} \varepsilon_{0}\right)}{D}}\left[x-2 \sqrt{D r_{m}\left(r_{m} \varepsilon_{0}+1\right)} t\right]\right)},
$$

which is plotted in Figure 5 and compared with numerical integration of the dynamic equations. The other cases are straightforward.

\section{CONCLUSIONS}

Variations in temperature and other climatic factors can produce environmental fluctuations that can modify the invasion patterns of populations. The question of how such external stochastic factors affect population dynamics will become more crucial with the expected increase in climatic variability (Schreiber and Ryan, 2011). We have obtained the density profile and the invasion velocity in the presence of such external fluctuations for reaction-diffusion models of population growth. We have considered the situations where they give rise to noise in the density-independent growth rate, the competition term or the Allee threshold. We have been able to address this problem in an analytically tractable model by reducing the SPDE to an effective deterministic equation for the mean density that incorporates the systematic effect of the noise. We have constructed a variational principle that is able to provide the mean density profile and the invasion velocity for both pulled and pushed fronts.

If fluctuations affect the density-independent growth rate, the mean population density of the invaded area and the invasion velocity experience an increase with the noise intensity. Both increases have exactly the same cause, namely an increase in the effective density-independent growth rate due to 
the systematic contribution of the multiplicative external noise. This result differs from previous studies that either considered intrinsic stochastic factors or adopted discrete-time integrodifference descriptions of population growth. Lewis analyzed the effect of internal fluctuations in a discrete-time model (Lewis, 2000). He found, in agreement with a long-time conjecture in the literature, that "intrinsic stochastic factors associated with interacting individuals can slow the spread of a population or disease, even in a uniform environment." Schreiber and Ryan employed an integrodifference matrix population model to investigate the effect of external stochastic factors and also found that increased variability in vital rates leads to slower invasion speeds (Schreiber and Ryan, 2011). Clearly, the nature of the fluctuations, intrinsic versus external, plays an important role for the invasion speed. So does the nature of the dynamics, discrete-time integrodifference equations versus continuous-time reaction-diffusion equations.

If fluctuations affect the competition term, two different fronts travelling with different velocities appear depending on the initial conditions. One corresponds to the deterministic invasion front. (The initial conditions for this type of front correspond to the usual conditions of a non-native species being introduced locally at small to moderate densities.) Here the noise raises the carrying capacity of the system, while having no effect on the invasion speed. The other front represents a noise-induced phenomenon, namely the "collapse" of a noise-sustained high-density state. (The initial conditions for this type of front correspond to a high-density state that arose due to some catastrophic event triggering a sudden explosive growth in the population.) The front speed is strongly dependent on the noise strength and decreases with increasing noise amplitude. In other words, stronger noise extends the lifetime of the noise- 
induced high density state.

In the case of logistic growth, the systematic effect of the external noise in either the coefficient of increase or the limiting coefficient does not change the pulled character of the front. If the population experiences the Allee effect, the invasion front is pushed in the absence of noise. In this case, the invasion velocity cannot be calculated by the linear conjecture, because the front dynamics is governed by the nonlinear terms. However, in the presence of fluctuations in the Allee threshold, both pulled and pushed fronts are possible, and we have been able to predict analytically the transition diagram. The dynamics of the system is very rich and the velocity and success or failure of the invasion depends on the balance between the average Allee threshold and the noise strength. Our results agree with numerical simulations and can be generalized to two dimensional invasions and the case of colored noises.

Our results for the logistic growth model show that fluctuations in the densityindependent growth rate are more "dangerous" than fluctuations in the competition term. The former increase the velocity of invasion, while the latter have no effect. This suggests that if the effects of external variability on the vital rates of a non-native species can be mitigated, then the effort should be directed towards minimizing the noise strength of the density-independent growth rate. It should be possible to test our predictions in experiments of the type conducted by Melbourne and Hastings. The authors were interested in the effects of endogenous variability on invasion velocity in those experiments and tightly controlled the ambient environment (Melbourne and Hastings, 2009). It should be possible to repeat the experiments and introduce deliberate and controlled variability of the environment. A comparison of the two types of experiments should reveal the effects of external fluctuations on the invasion 
velocity.

\section{Acknowledgments}

The work has been funded by Direcció General de Recerca-Generalitat de Catalunya under grant 2009 SGR-164 (VM and DC) and by MICINN Grant No. FIS 2006-12296-C02-01 (VM).

\section{Appendix A. Mean value of the noise term}

The mean value can be evaluated as follows:

$$
\begin{aligned}
\langle g(\phi) \eta(x, t)\rangle & =\int_{0}^{t} d t^{\prime} \int d x^{\prime}\left\langle\eta(x, t) \eta\left(x^{\prime}, t^{\prime}\right)\right\rangle\left\langle\frac{\delta g(\phi(x, t))}{\delta \eta\left(x^{\prime}, t^{\prime}\right)}\right\rangle \\
& =2 \int d x^{\prime} C\left(\left|x-x^{\prime}\right| / \lambda\right)\left\langle\left.\frac{d g(\phi)}{d \phi} \frac{\delta \phi(x, t)}{\delta \eta\left(x^{\prime}, t^{\prime}\right)}\right|_{t^{\prime}=t}\right\rangle .
\end{aligned}
$$

Equation (4) can be integrated formally to obtain

$$
\begin{aligned}
\phi(x, t) & =\phi(x, 0)+D \frac{\partial^{2}}{\partial x^{2}} \int_{0}^{t} d s \phi(x, s)+\int_{0}^{t} d s F[\phi(x, s)] \\
& +\varepsilon^{1 / 2} \int_{0}^{t} d s g[\phi(x, s)] \eta(x, s)
\end{aligned}
$$

and (38) implies that

$$
\frac{\delta \phi(x, t)}{\delta \eta\left(x^{\prime}, t^{\prime}\right)}=\varepsilon^{1 / 2} \int_{0}^{t} d s g[\phi(x, s)] \frac{\delta \eta(x, s)}{\delta \eta\left(x^{\prime}, t^{\prime}\right)}=\varepsilon^{1 / 2} \delta\left(x^{\prime}-x\right) g\left[\phi\left(x, t^{\prime}\right)\right] .
$$

Substituting (39) into (37), we obtain 


$$
\begin{aligned}
\langle g(\phi) \eta(x, t)\rangle & =2 \varepsilon^{1 / 2}\left\langle\frac{d g(\phi)}{d \phi} g(\phi)\right\rangle \int d x^{\prime} C\left(\left|x-x^{\prime}\right| / \lambda\right) \delta\left(x^{\prime}-x\right) \\
& =\varepsilon^{1 / 2} C(0)\left\langle\frac{d g(\phi)}{d \phi} g(\phi)\right\rangle
\end{aligned}
$$

where in the last equality we have made use of the the Stratonovich interpretation of stochastic calculus $\left(\int d x^{\prime} \delta\left(x^{\prime}-x\right)=1 / 2\right)$. Equation (40) is Novikov's theorem for spatially extended systems (Novikov, 1965).

\section{Appendix B. Variational principle}

In order to formulate a variational principle we start with the reaction-diffusion equation

$$
\frac{\partial \rho(x, t)}{\partial t}=D \frac{\partial^{2} \rho(x, t)}{\partial x^{2}}+F(\rho)
$$

The time evolution of an initial condition $\rho(x, 0)$ has been studied both for $F(\rho)$ monostable or bistable. It was proven (Aronson and Weinberger, 1978) that suitable initial conditions evolve into a monotonic traveling front $\rho(x-v t)$, joining the stable state $\rho=1$ to $\rho=0$. If $F$ is monostable, e.g., logistic growth, there exists a continuum of values of $v$ for which a monotonic (pulled) front occurs. If the initial condition has compact support, the front with minimal velocity is selected. If $F$ is bistable, e.g., growth with Allee effect, there is a single isolated value of the velocity for which the (pushed) front exists. In this appendix we show how to derive the velocity for pushed fronts and the minimal velocity in the case of pulled fronts from a variational principle following the method developed by Benguria and Depassier, 1996.

To this end, consider (41) transformed to the frame commoving with the front

$$
D \frac{d^{2} \rho}{d z^{2}}+v \frac{d \rho}{d z}+F(\rho)=0
$$


where $v$ is the asymptotic front velocity and $z=x-v t$ is the commoving coordinate. For simplicity, we assume that the front connects the state $\rho=0$ (unstable) to the state $\rho=1$ (stable). One can define the front slope as $p(\rho)=-d \rho / d z$, which is a positive function for every value of the density. Therefore, (42) can be written as,

$$
D p \frac{d p}{d \rho}-v p+F(\rho)=0
$$

with the conditions $p(0)=p(1)=0$. Defining $g(\rho)$ as a positive trial function on $(0,1)$, such that $h=-d g / d \rho>0$, multiplying (43) by $g(\rho)$, and integrating by parts we obtain

$$
\int_{0}^{1} F g d \rho=\int_{0}^{1}\left(v p g-\frac{1}{2} D h p^{2}\right) d \rho
$$

For fixed $\rho$, the functional

$$
\Phi[p] \equiv v p g-\frac{1}{2} D h p^{2}
$$

has a maximum at $p_{\max }=v g /(D h)$, since $v, p, g$ and $h$ are positive. Therefore $\Phi[p] \leq \Phi\left[p_{\max }\right]=v^{2} g^{2} /(2 D h)$ for any value of $\rho$. Equations (44) and (45) lead to the following condition, which is valid for any reaction function $F(\rho)$ :

$$
v^{2} \geq 2 D \frac{\int_{0}^{1} F g d \rho}{\int_{0}^{1}\left(g^{2} / h\right) d \rho} .
$$

The equality in (46) holds if

$$
-\frac{d}{d \rho}\left(\ln g^{*}\right)=\frac{v}{D p}
$$

which can be integrated to yield

$$
g^{*}(\rho)=\exp \left(\int_{\rho}^{\rho_{0}} \frac{v}{D p} d \rho\right)
$$


where $0<\rho_{0}<1$ comes from the integration constant. Obviously, $g^{*}(\rho)$ is a continuous, positive and decreasing function on $(0,1)$ with $g^{*}(1)=0$. Near $\rho=0, g^{*}$ diverges. We linearize (43) to find from (48) that $g^{*}$ diverges as

$$
g^{*}(\rho) \sim \rho^{-v /\left(D \mu_{+}\right)}
$$

near $\rho=0$, with $\mu_{+}=\left[v+\sqrt{v^{2}-4 D F^{\prime}(0)}\right] / 2 D$. Consequently, $F g \sim \rho g \sim$ $\rho^{1-v /\left(D \mu_{+}\right)}$near $\rho=0$. Analogously $g^{2} / h \sim \rho^{1-v /\left(D \mu_{+}\right)}$near $\rho=0$, so the integrals in (46) exist if $v /\left(D \mu_{+}\right)<2$. Since this condition is always satisfied, we can ensure that the equality in (46) is well established. Thus

$$
v^{2}=\max _{g}\left[2 D \frac{\int_{0}^{1} F g d \rho}{\int_{0}^{1}\left(g^{2} / h\right) d \rho}\right],
$$

where the maximum is taken over all positive, decreasing trial functions on $(0,1)$. Since (49) implies that $g$ must behave like $\rho^{-\lambda}$, with $\lambda>0$, near $\rho=0$ and must be such that $g(1)=0$, we can choose the trial function $g(\rho)=$ $\rho^{-\lambda}(1-\rho)^{\lambda}$. With this choice, (50) has to be maximized over the parameter $\lambda$, but only for values such that the integrals in the variational principle exist,

$$
v^{2}=2 D \max _{0<\lambda \leq 2}\left[\frac{\lambda \Gamma(4)}{\Gamma(2-\lambda) \Gamma(2+\lambda)} \int_{0}^{1} F(\rho) \rho^{-\lambda}(1-\rho)^{\lambda} d \rho\right] .
$$

To confirm that the variational formula provides the correct front velocities, we apply it to a simple case. If the reaction term is logistic, $F(\rho)=r \rho(1-\rho)$, without noise, the above equation leads to the Fisher front velocity,

$$
v \equiv v_{F}=2 \sqrt{D r}
$$




\section{Appendix C. Numerical algorithm}

The numerical integration of (15), (17) and (19) was performed using the general approach for SPDE (San Miguel and Toral, 2000). The habitat is simulated as a grid consisting of $N$ lattice sites of size $\Delta x$, such that $L=N \Delta x$ is the habitat size. Every lattice site $i$ is identified by its position $x_{i}$, which is a discrete variable. The continuum density, $\phi(x, t)$, is replaced by its analogue in discrete space, $\phi\left(x_{i}, t\right)$, and the external noise is simulated via the generation of Gaussian numbers, where we replace the white noise $\eta(x, t)$ by $\frac{1}{\sqrt{\Delta x}} \eta_{i}(t)$, as a result of the relation between the Dirac and the Kronecker delta functions. The correlation function is given by $\left\langle\eta_{i}(t) \eta_{j}\left(t^{\prime}\right)\right\rangle=\delta_{i j} \delta\left(t-t^{\prime}\right)$. Moreover, the noise amplitude is $\varepsilon C(0)=\varepsilon / \Delta x$ (Santos and Sancho, 1999).

Equations (15), (17) and (7) can be written as,

$$
\frac{\partial \phi}{\partial t}=D \frac{\partial^{2} \phi}{\partial x^{2}}+F(\phi)+G(\phi) \eta
$$

where $G(\phi)=\varepsilon^{1 / 2} g(\phi)$. Assuming uniform time steps of size $\Delta t$ and integrating (15) and (17) using the Euler algorithm, we obtain a dynamic equation that is discrete in both space and time,

$$
\begin{aligned}
\phi_{t+\Delta t, i} & =\phi_{t, i}+\Delta t \frac{D}{\Delta x^{2}}\left(\phi_{i+1, t}-2 \phi_{i, t}+\phi_{i-1, t}\right)+\Delta t^{1 / 2} G\left(\phi_{i, t}\right) u_{t, i} \\
& +\Delta t\left[F\left(\phi_{i, t}\right)+\frac{1}{2} G\left(\phi_{i, t}\right) G^{\prime}\left(\phi_{i, t}\right) u_{t, i}^{2}\right]
\end{aligned}
$$

where $\Delta t^{1 / 2} u_{t, i}=\eta_{i}(t)$. Simulations have been performed with $\Delta x=0.1$, $\Delta t=0.0025$ and $D=1$. We have varied the parameters $r, K, \varepsilon$ and $L$ in different simulations. 


\section{References}

Allee, W.C., 1938. The Social Life of Animals. Heinemann, London, pp.72-111.

Armero, J., Sancho, J.M., Casademunt, J., Lacasta, A.M., Ramirez-Piscina, L., Sagués, F., 1996. External Fluctuations in Front Propagation. Phys. Rev. Lett. 76, 3045-3048.

Aronson, D.G., Weinberger, H.F., 1978. Multidimensional nonlinear diffusion arising in population genetics. Advances in Mathematics 30, 33-76.

Benguria, R.D., Depassier, M.C., 1996. Speed of fronts of the reaction-diffusion equation. Physical Review Letters 77, 1171-1174.

Feldman, M.W., Roughgarden, J., 1975. A population's stationary distribution and chance of extinction in a stochastic environment with remarks on the theory of species packing. Theor. Pop. Biol. 7, 197-207.

Fisher, R. A., 1937. The Wave of Advance of Advantageous Genes. Annals of Eugenics 7, 355-369

García-Ojalvo, J., Sancho, J.M., 1999. Noise in Spatially Extended Systems. Springer-Verlag, New York.

Hengeveld, R., 1989. Dynamics of Biological Invasions. Chapman and Hall, London.

Hizanidis, J., Balanov, A., Amann, A., Schöll, E., 2006. Noise-Induced Front Motion: Signature of a Global Bifurcation. Phys. Rev. Lett. 96, 244104.

Horsthemke, W., Lefever, R., 1984. Noise-Induced Transitions. Springer-Verlag, 
Berlin.

Kolmogorov, A. N., Petrovsky, I. G., Piskunov, N. S., 1937. Study of the equation of diffusion equation combined with an increase of matter and its application to a biological problem. Bulletin of Moscow State University, 1, $1-25$.

Lewis, M. A., 2000, Spread rate for a nonlinear stochastic invasion. J. Math. Biol. 41, 430-454.

Malchow, H., Hilker, F. M., Petrovskii, S. V., 2004a. Noise and productivity dependence of spatiotemporal pattern formation in a prey-predator system. Discrete and Continuous Dynamical Systems B, 4, 705-711.

Malchow, H., Hilker, F. M., Petrovskii, S. V., Brauer, K., 2004b. Oscillations and waves in a virally infected plankton system Part I: The lysogenic stage. Ecological Complexity, 1, 211-223.

Marcus, R., 1982. A stochastic logistic diffusion equation. Mathematical Biosciences $62,281-294$.

Melbourne, B.A., Hastings, A. 2008. Extinction risk depends strongly on factors contributing to stochasticity. Nature 454, 100-103.

Melbourne, B. A., Hastings, A., 2009. Highly variable spread rates in replicated biological invasions: fundamental limits to predictability. Science, 325, 15361539.

Méndez, V., Compte, A., 1998. Wavefronts in bistable hyperbolic reactiondiffusion systems. Physica A 260, 90-98. 
Méndez, V., Fedotov, S., Horsthemke, W., 2010. Reaction-Transport Systems.

Mesoscopic Foundations, Fronts and Spatial Instabilities. Springer-Verlag, Berlin.

Murray, J.D., 2003. Mathematical Biology. Springer, New York.

Novikov, E.A., 1965. Functionals and the random-force method in turbulence theory (Euler velocity field described by random forces method, using Lagrangian representation of turbulence). Soviet Physics JETP 20, 1290-1294.

Ortega-Cejas, V., Fort, J., Méndez, V., 2004. Role of the delay time in the modelling of biological range expansions. Ecology 85, 258-264.

San Miguel, M., Toral, R., 2000. Instabilities and Nonequilibrium Structures VI. Kluwer Academic Publishers, pp 35-130.

Santos, M.A., Sancho, J.M., 1999. Noise-induced fronts. Physical Review E $59,98-102$.

Scarsoglio, S., Laio, F., D’Odorico, P., Ridolfi, L., 2011. Spatial pattern formation induced by Gaussian white noise. Math. Biosci. 229, 174-184

Schimansky-Geier, L., Zülicke, C., 1991. Kink propagation induced by multiplicative noise. Z. Phys. B 82, 157-162.

Shigesada, N., Kawasaki, K., 1997. Biological Invasions: Theory and Practice. Oxford University Press, Oxford.

Skellam, J. G., 1951. Random Dispersal in Theoretical Populations. Biometrika, $38,196-218$

Tuckwell, H.C., 1974. A study of some diffusion models of population growth. Theoretical Population Biology 5, 345-357. 
van Saarloos, W., 2003. Front propagation into unstable states. Physics Reports $386,29-222$.

Williamson, M., 1996. Biological Invasions. Chapman and Hall, London. 


\section{Figure captions}

Figure 1.

Invasion velocity as a function of the noise amplitude if $r_{1}$ fluctuates; $C(0)=$ $10, r_{m}=0.1, D=1$. Solid lines correspond to the analytical expression (22) and circles to numerical solutions. Inset: Logarithmic plot to show that the dependence of the velocity on the noise amplitude is square root like (slope $1 / 2)$.

\section{Figure 2.}

Invasion velocity as a function of the noise amplitude if $K$ fluctuates, with $K_{m}=1$. Solid lines correspond to the analytical expression (28) and circles to numerical solutions. a) Invasion velocity for a front that connects $\rho_{-}$with $\rho=0$. b) Invasion velocity for a front that connects $\rho_{-}$with $\rho_{+} \cdot C(0)=10$, $r=0.1, D=1$. In both cases $x_{0}=10$.

Figure 3.

Diagram of the different invasion front regimes if the Allee threshold fluctuates. Solid lines correspond to the analytical expressions and circles to numerical solutions.

Figure 4.

Invasion velocity for a front connecting $\rho=0$ to $\rho=1$ (region I). The boundary between the regions of pulled and pushed fronts occurs at $r \varepsilon_{0}=6 ; a_{m}=1 / 4$. The initial condition taken is $\rho(x, 0)=1-\theta(x)$.

Figure 5.

Front profiles at different times for a fluctuating intrinsic net growth rate; $C(0)=10, r_{m}=0.1, D=1$ and $L=5000$. The final stable state is $\rho_{0}=$ $1+r_{m} \varepsilon_{0}=2$. Analytical and numerical results are plotted. The analytical profile calculated from (36) is drawn for $t=500$ and $\rho_{*}=4 / 3$. 


\section{ACCEPTED MANUSCRIPT}

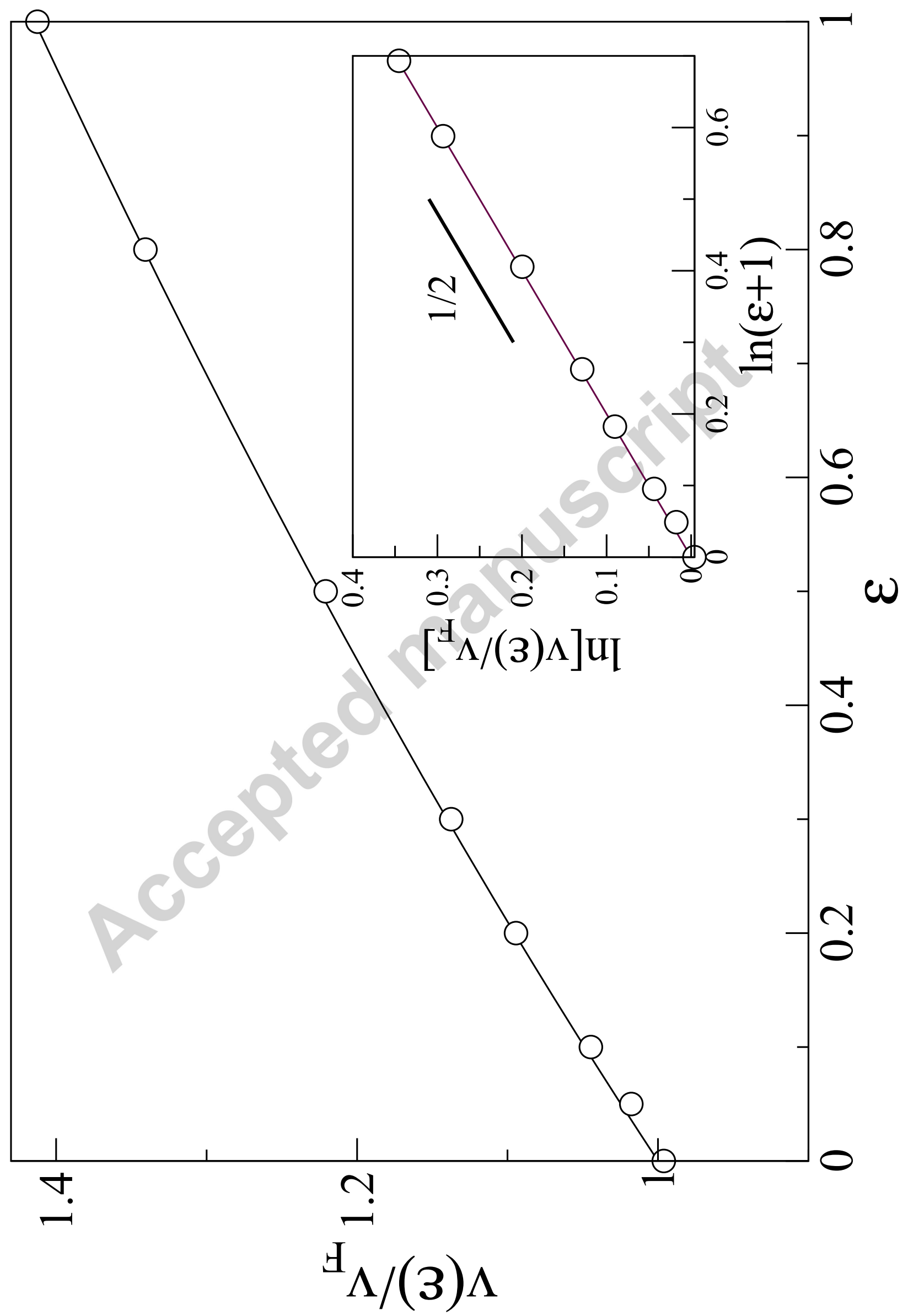



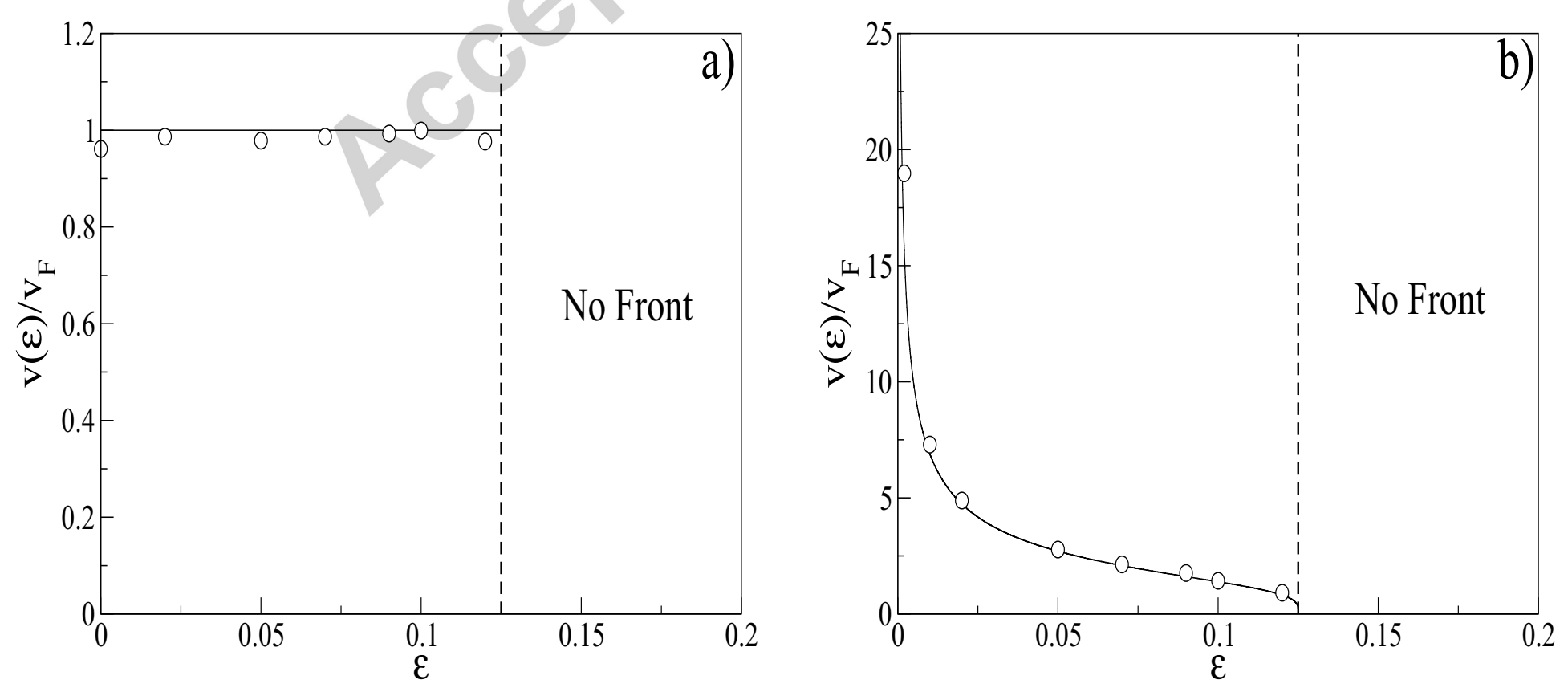


\section{ACCEPTED MANUSCRIPT}

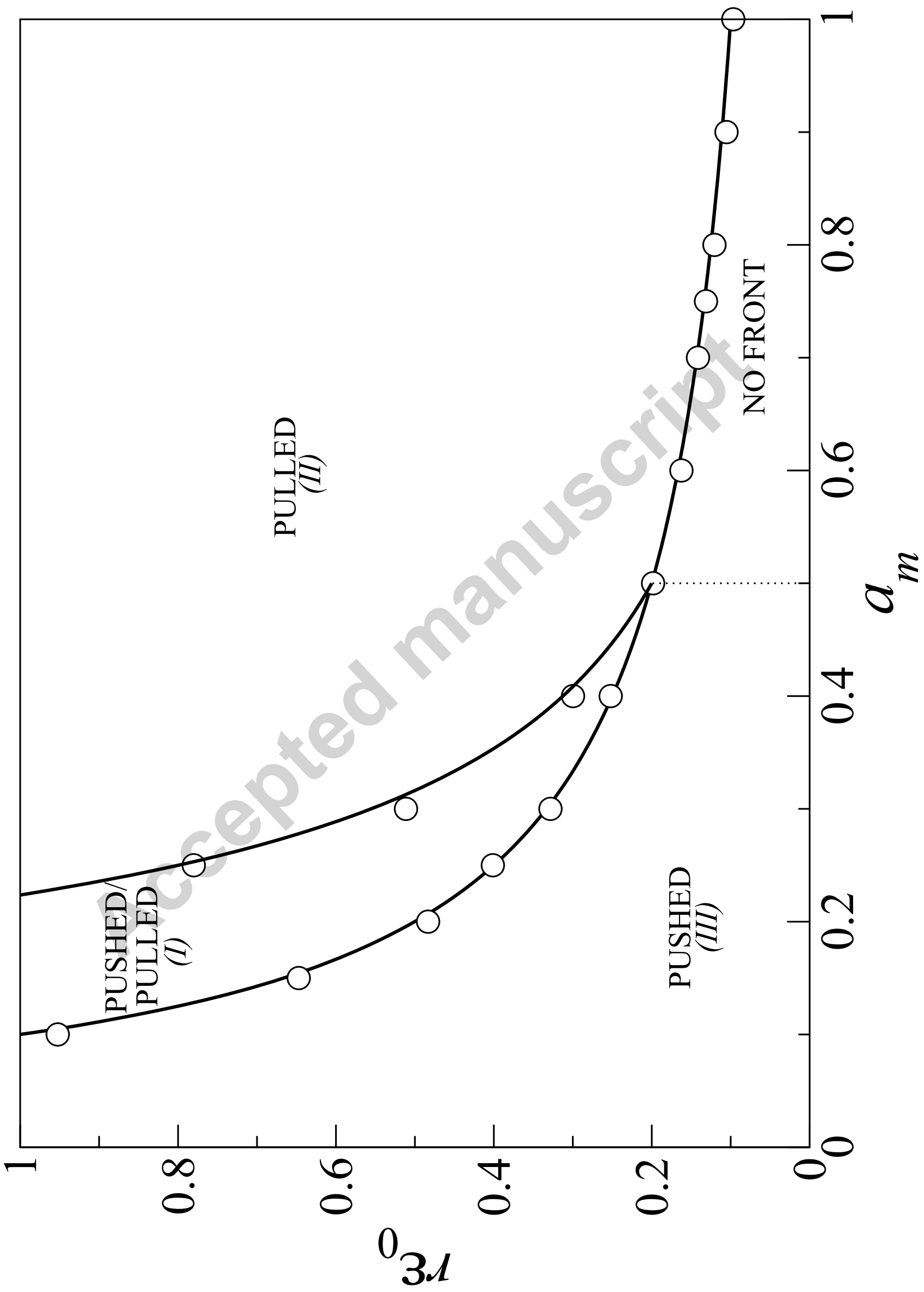




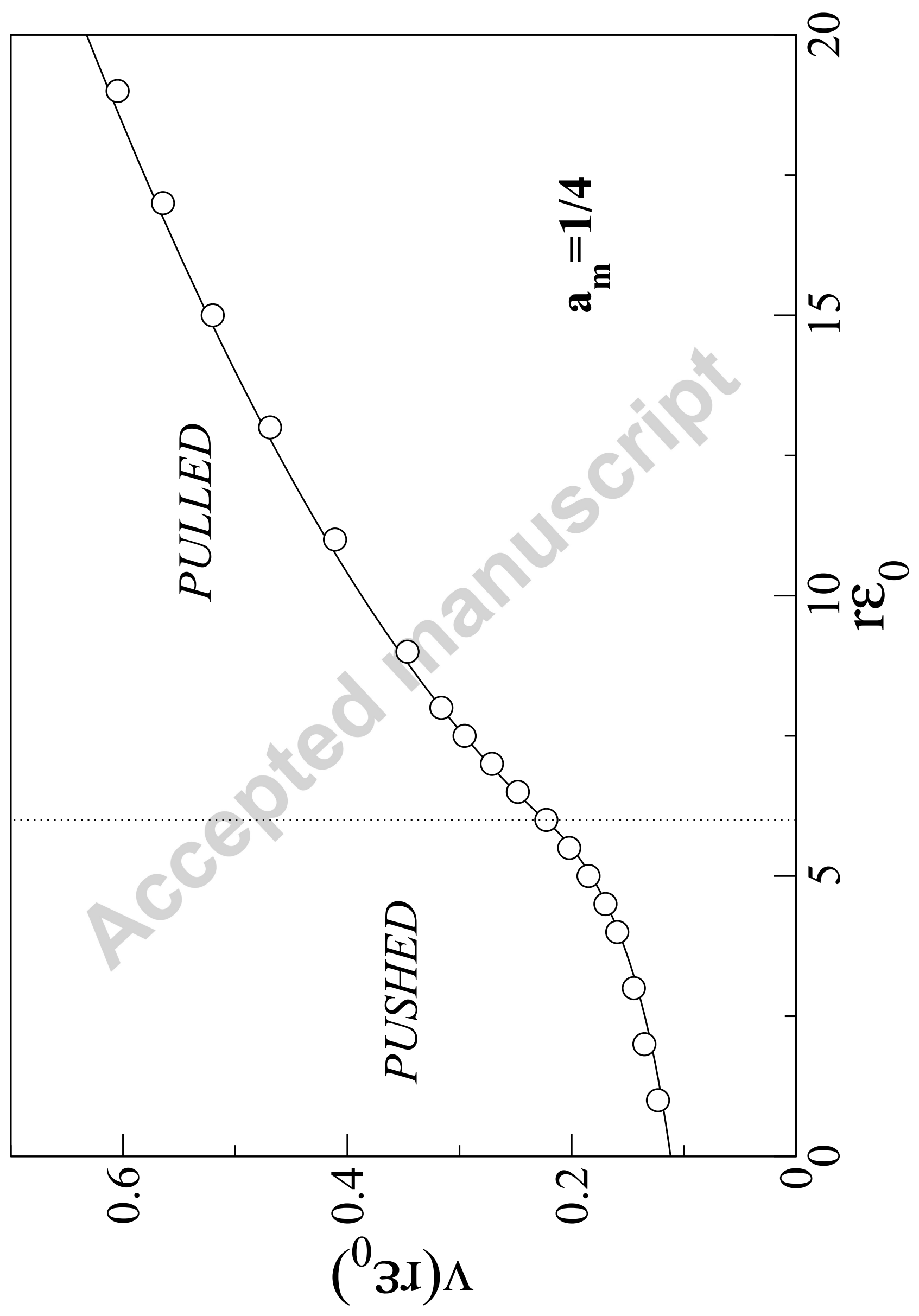




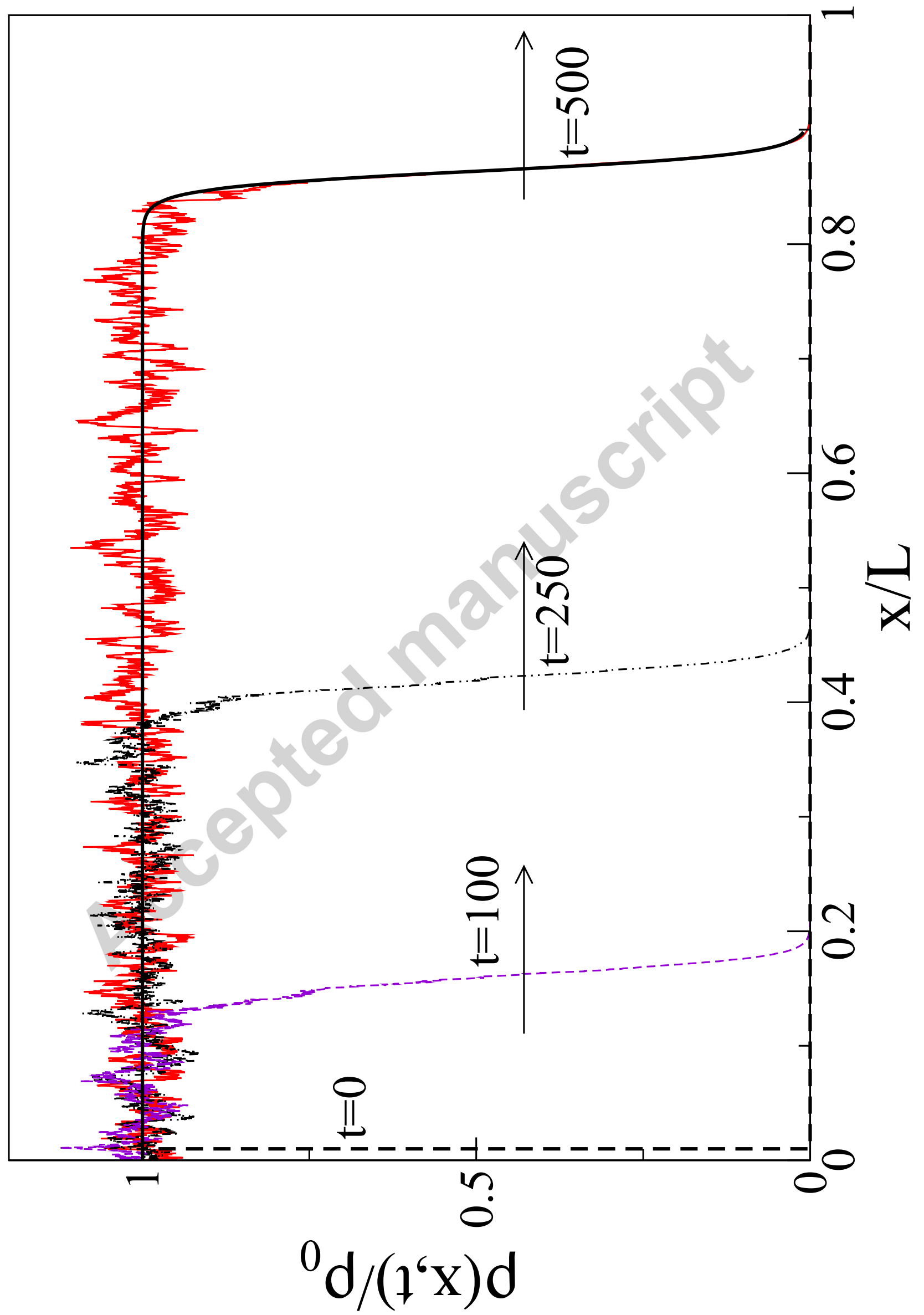


Highlights

$>$ We determine invasion front velocity in presence of environmental fluctuations

$>$ Noise is included in growth rate, intraspecific competition term and Allee threshold

$>$ Noise in the Allee threshold can induce pulled invasion fronts and invasion failure 